\title{
An Inequality for Gaussians on Lattices
}

\author{
Oded Regev*† \\ Noah Stephens-Davidowitz ${ }^{*}$ \\ noahsdecs.nyu. edu
}

\begin{abstract}
We show that for any lattice $\mathcal{L} \subseteq \mathbb{R}^{n}$ and vectors $\mathbf{x}, \mathbf{y} \in \mathbb{R}^{n}$,$$
\rho(\mathcal{L}+\mathbf{x})^{2} \rho(\mathcal{L}+\mathbf{y})^{2} \leq \rho(\mathcal{L})^{2} \rho(\mathcal{L}+\mathbf{x}+\mathbf{y}) \rho(\mathcal{L}+\mathbf{x}-\mathbf{y}),
$$

where $\rho$ is the Gaussian mass function $\rho(A):=\sum_{\mathbf{w} \in A} \exp \left(-\pi\|\mathbf{w}\|^{2}\right)$. We show a number of applications, including bounds on the moments of the discrete Gaussian distribution, various monotonicity properties of the heat kernel on flat tori, and a positive correlation inequality for Gaussian measures on lattices.
\end{abstract}

\section{Introduction}

A lattice $\mathcal{L} \subset \mathbb{R}^{n}$ is the set of all integer linear combinations of $n$ linearly independent vectors $\mathbf{B}=\left(\mathbf{b}_{1}, \ldots, \mathbf{b}_{n}\right)$. For any $s>0$, we define the function $\rho_{s}: \mathbb{R}^{n} \rightarrow \mathbb{R}$ as

$$
\rho_{s}(\mathbf{x})=\exp \left(-\pi\|\mathbf{x}\|^{2} / s^{2}\right) .
$$

For a discrete set $A \subset \mathbb{R}^{n}$ we define $\rho_{s}(A)=\sum_{\mathbf{w} \in A} \rho_{s}(\mathbf{w})$. The discrete Gaussian distribution over a lattice coset $\mathcal{L}+\mathbf{x}$ with parameter $s, D_{\mathcal{L}+\mathbf{x}, s}$ is the probability distribution over $\mathcal{L}+\mathbf{x}$ that assigns probability

$$
\frac{\rho_{s}(\mathbf{w})}{\rho_{s}(\mathcal{L}+\mathbf{x})}
$$

to each vector $\mathbf{w} \in \mathcal{L}+\mathbf{x}$. (See Figure $1 \mathrm{a}$.) The periodic Gaussian function over $\mathcal{L}$ with parameter $s$ is

$$
f_{\mathcal{L}, s}(\mathbf{x}):=\frac{\rho_{s}(\mathcal{L}+\mathbf{x})}{\rho_{s}(\mathcal{L})}
$$

(See Figure $1 \mathrm{~b}$,) When $s=1$, we write $\rho(\mathbf{x}), D_{\mathcal{L}+\mathbf{x}}$, and $f_{\mathcal{L}}(\mathbf{x})$.

These objects appear in several guises in mathematics and are well studied. For example, $\rho(\mathcal{L}+\mathbf{x})$ is the Riemann theta function in a dual form (see, e.g., [Mum07]) and was studied in

*Courant Institute of Mathematical Sciences, New York University.

${ }^{+}$Supported by the Simons Collaboration on Algorithms and Geometry and by the National Science Foundation (NSF) under Grant No. CCF-1320188. Any opinions, findings, and conclusions or recommendations expressed in this material are those of the authors and do not necessarily reflect the views of the NSF.

‡Supported by the National Science Foundation (NSF) under Grant No. CCF-1320188. Any opinions, findings, and conclusions or recommendations expressed in this material are those of the authors and do not necessarily reflect the views of the NSF. 


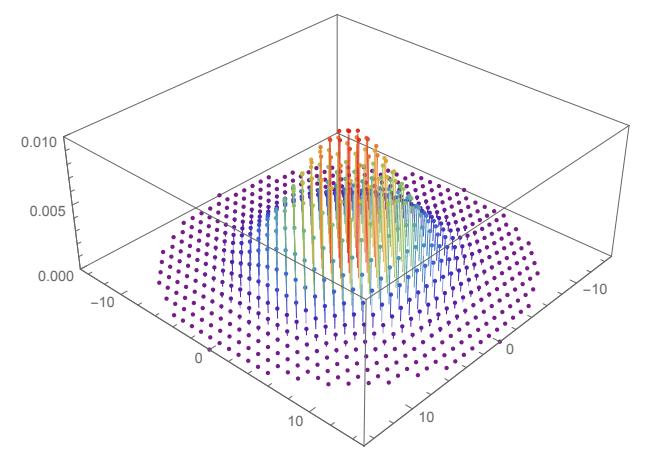

(a) The discrete Gaussian distribution on $\mathbb{Z}^{2}$ with parameter $s=10$.

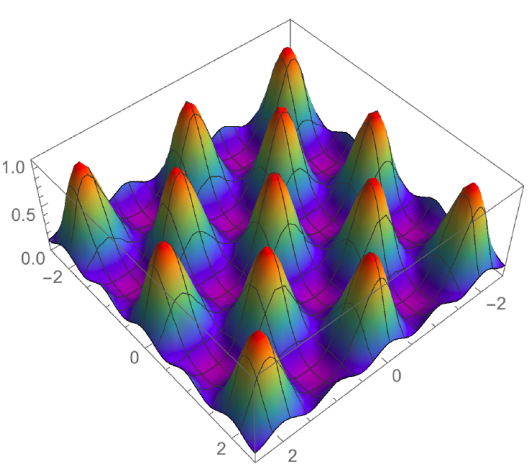

(b) The periodic Gaussian function on the lattice spanned by $(1,1)$ and $(1,-1)$ with parameter $s=3 / 4$.

Figure 1

connection with the Riemann zeta function [Rie57, BPY01]; it can also be seen as the heat kernel on the flat torus $\mathbb{R}^{n} / \mathcal{L}$; it played an instrumental role in proving tight transference theorems for lattices [Ban93]; it was used to construct bilipschitz embeddings of flat tori into a Hilbert space [HR13]; and the authors recently used it to bound the number of short lattice points [RS16b]. Both $D_{\mathcal{L}+\mathbf{x}}$ and $f_{\mathcal{L}}$ have also played an important role in recent years in computer science, especially in cryptographic applications of lattices (e.g., [MR07, GPV08]). Our motivation comes from attempts to improve upon the current fastest known algorithms for the main computational problems on lattices, the Shortest Vector Problem [ADRS15] and the Closest Vector Problem [ADS15]. (Both algorithms rely on special cases of the main result of this work.)

In spite of their importance, there is still a lot that we do not know about $\rho_{S}(\mathcal{L}+\mathbf{x}), f_{\mathcal{L}, s}(\mathbf{x})$, and $D_{\mathcal{L}+\mathbf{x}, s}$. In this work, we prove several basic inequalities concerning these objects, as described below. All of these inequalities follow without too much effort from one main inequality (Theorem 2.1), which is closely related to Riemann's theta relations (see [Mum07]). Namely, in terms of the periodic Gaussian $f_{\mathcal{L}}(\mathbf{x})$, our main inequality says that

$$
f_{\mathcal{L}}(\mathbf{x})^{2} f_{\mathcal{L}}(\mathbf{y})^{2} \leq f_{\mathcal{L}}(\mathbf{x}+\mathbf{y}) f_{\mathcal{L}}(\mathbf{x}-\mathbf{y}) .
$$

Note that the Gaussian function $\rho(\mathbf{x})$ over $\mathbb{R}^{n}$ satisfies the "rotation" identity

$$
\rho(\mathbf{x})^{2} \rho(\mathbf{y})^{2}=\rho(\mathbf{x}+\mathbf{y}) \rho(\mathbf{x}-\mathbf{y}),
$$

so that our main inequality can be viewed as a relaxation of this identity to the periodic case. From this (perhaps rather opaque) inequality, we derive many natural statements concerning $\rho_{\mathcal{S}}(\mathcal{L}+\mathbf{x})$, $f_{\mathcal{L}, s}(\mathbf{x})$, and $D_{\mathcal{L}+\mathbf{x}, s}$.

First, we show in Corollary 3.2 that the covariance of $D_{\mathcal{L}+\mathbf{x}}$ is minimized when $\mathbf{x}=\mathbf{0}$, answering a natural question communicated to us by Dadush [Dad13]. (We note in passing that closely related questions are still open, e.g., whether $\mathbb{E}_{\mathbf{w} \sim D_{\mathcal{L}+\mathbf{x}}}[\|\mathbf{w}\|]$ is minimized when $\mathbf{x}=\mathbf{0}$.) Along the way, we derive an interesting inequality concerning the "shape" of $f_{\mathcal{L}}(\mathbf{x})$ (Proposition 3.1). We also analyze the fourth moment, showing in particular that the discrete Gaussian is "leptokurtic" (Proposition 3.3) - i.e., its kurtosis is at least that of the continuous Gaussian distribution. 
Second, in Section 4, we show various monotonicity results concerning $f_{\mathcal{L}, s}$, answering a natural open question due to Price [Pri14b] in the affirmative. In particular, in Proposition 4.1 we show that $f_{\mathcal{L}, s}$ is monotonic in $s$ and in Proposition 4.2 , we extend this to the non-spherical Gaussian case. (Recently, Price showed how to derive from this an analogous monotonicity result for Abelian Cayley graphs [Pri16]. A further extension to arbitrary Cayley graphs, previously suggested by Peres [Per13], turns out to be false [RS16a].) Additionally, in Proposition 4.3, we show that $f_{\mathcal{L}, s}$ is monotonic under taking sublattices of $\mathcal{L}$.

Finally, in Section 5, we show that sublattices of a lattice $\mathcal{L}$ are positively correlated under the normalized Gaussian measure on $\mathcal{L}$. This result answers another open question due to Price [Pri14a], and was recently used by him in his work on cohomology [Pri15]. It has a (possibly superficial) resemblance to the recently proven Gaussian correlation conjecture on symmetric convex bodies [Roy14]. In fact, we note in passing that our main inequality can also be viewed as a correlation result. In particular, it shows that $\cos (2 \pi\langle\mathbf{w}, \mathbf{x}\rangle)$ and $\cos (2 \pi\langle\mathbf{w}, \mathbf{y}\rangle)$ are positively correlated when $\mathbf{w}$ is sampled from $D_{\mathcal{L}}$. (See Eq.4e.)

\section{The main inequality}

The following is our main theorem. The proof is essentially a combination of a certain identity related to Riemann's theta relations (see [Mum07, Chapter 1, Section 5]) and the Cauchy-Schwarz inequality.

Theorem 2.1. For any lattice $\mathcal{L} \subset \mathbb{R}^{n}$ and any two vectors $\mathbf{x}, \mathbf{y} \in \mathbb{R}^{n}$, we have

$$
\rho(\mathcal{L}+\mathbf{x})^{2} \rho(\mathcal{L}+\mathbf{y})^{2} \leq \rho(\mathcal{L})^{2} \rho(\mathcal{L}+\mathbf{x}+\mathbf{y}) \rho(\mathcal{L}+\mathbf{x}-\mathbf{y}) .
$$

Proof. Let $\mathcal{L}^{\oplus 2}:=\mathcal{L} \oplus \mathcal{L}$. We can then write $\rho(\mathcal{L}+\mathbf{x}) \rho(\mathcal{L}+\mathbf{y})=\rho\left(\mathcal{L}^{\oplus 2}+(\mathbf{x}, \mathbf{y})\right)$. Consider the $2 n \times 2 n$ matrix

$$
T:=\left(\begin{array}{rr}
I_{n} & I_{n} \\
I_{n} & -I_{n}
\end{array}\right)
$$

where $I_{n}$ is the $n \times n$ identity matrix. Note that $T / \sqrt{2}$ is an orthogonal matrix so that $\|T \mathbf{v}\|=$ $\sqrt{2}\|\mathbf{v}\|$ for any $\mathbf{v} \in \mathbb{R}^{2 n}$. We therefore have

$$
\rho(\mathcal{L}+\mathbf{x}) \rho(\mathcal{L}+\mathbf{y})=\rho_{\sqrt{2}}\left(T\left(\mathcal{L}^{\oplus 2}+(\mathbf{x}, \mathbf{y})\right)\right)=\rho_{\sqrt{2}}\left(T \mathcal{L}^{\oplus 2}+(\mathbf{x}+\mathbf{y}, \mathbf{x}-\mathbf{y})\right) .
$$

For any $\mathbf{z}:=\left(\mathbf{z}_{1}, \mathbf{z}_{2}\right) \in \mathcal{L}^{\oplus 2}$, we have $T \mathbf{z}=\left(\mathbf{w}_{1}, \mathbf{w}_{2}\right)$ where $\mathbf{w}_{1}:=\mathbf{z}_{1}+\mathbf{z}_{2}$ and $\mathbf{w}_{2}:=\mathbf{w}_{1}-2 \mathbf{z}_{2}$. It follows that

$$
\begin{aligned}
T \mathcal{L}^{\oplus 2} & =\left\{\left(\mathbf{w}_{1}, \mathbf{w}_{2}\right) \in \mathcal{L}^{2}: \mathbf{w}_{1} \equiv \mathbf{w}_{2} \bmod 2 \mathcal{L}\right\} \\
& =\bigcup_{\mathbf{c} \in \mathcal{L} /(2 \mathcal{L})}(2 \mathcal{L}+\mathbf{c})^{2}
\end{aligned}
$$

where the union is disjoint. Plugging in to Eq. (1), we have

$$
\rho(\mathcal{L}+\mathbf{x}) \rho(\mathcal{L}+\mathbf{y})=\sum_{\mathbf{c} \in \mathcal{L} /(2 \mathcal{L})} \rho_{\sqrt{2}}(2 \mathcal{L}+\mathbf{c}+\mathbf{x}+\mathbf{y}) \cdot \rho_{\sqrt{2}}(2 \mathcal{L}+\mathbf{c}+\mathbf{x}-\mathbf{y}) .
$$


Note that, by the right-hand side of $(2)$, we can view $\rho(\mathcal{L}+\mathbf{x}) \rho(\mathcal{L}+\mathbf{y})$ as the inner product of two vectors,

$$
\rho(\mathcal{L}+\mathbf{x}) \rho(\mathcal{L}+\mathbf{y})=\langle\mathbf{h}(\mathbf{x}+\mathbf{y}), \mathbf{h}(\mathbf{x}-\mathbf{y})\rangle
$$

where

$$
h(\mathbf{z}):=\left(\rho_{\sqrt{2}}\left(2 \mathcal{L}+\mathbf{z}+\mathbf{c}_{1}\right), \rho_{\sqrt{2}}\left(2 \mathcal{L}+\mathbf{z}+\mathbf{c}_{2}\right), \ldots, \rho_{\sqrt{2}}\left(2 \mathcal{L}+\mathbf{z}+\mathbf{c}_{2^{n}}\right)\right) \in \mathbb{R}^{2^{n}},
$$

for some ordering of the cosets $\mathbf{c}_{i} \in \mathcal{L} /(2 \mathcal{L})$. Then, by Cauchy-Schwarz, we have

$$
\rho(\mathcal{L}+\mathbf{x})^{2} \rho(\mathcal{L}+\mathbf{y})^{2} \leq\|\mathbf{h}(\mathbf{x}+\mathbf{y})\|^{2}\|\mathbf{h}(\mathbf{x}-\mathbf{y})\|^{2}=\rho(\mathcal{L})^{2} \rho(\mathcal{L}+\mathbf{x}+\mathbf{y}) \rho(\mathcal{L}+\mathbf{x}-\mathbf{y}),
$$

where the last equality follows from plugging in $\mathbf{y}=\mathbf{0}$ to Eq. (3) which tells us that $\|\mathbf{h}(\mathbf{z})\|^{2}=$ $\rho(\mathcal{L}) \rho(\mathcal{L}+\mathbf{z})$.

We remark that using the same proof with other transformations $T$ might lead to other such inequalities. We leave this for future work and proceed to list a few immediate corollaries of Theorem 2.1.

Corollary 2.2. For any lattice $\mathcal{L} \subset \mathbb{R}^{n}$ and any two vectors $\mathbf{x}, \mathbf{y} \in \mathbb{R}^{n}$, we have

$$
\begin{aligned}
f_{\mathcal{L}}(\mathbf{x})^{2} f_{\mathcal{L}}(\mathbf{y})^{2} & \leq f_{\mathcal{L}}(\mathbf{x}+\mathbf{y}) f_{\mathcal{L}}(\mathbf{x}-\mathbf{y}) \\
f_{\mathcal{L}}(\mathbf{x})^{4} & \leq f_{\mathcal{L}}(2 \mathbf{x}) \\
f_{\mathcal{L}}(\mathbf{x}) f_{\mathcal{L}}(\mathbf{y}) & \leq\left(f_{\mathcal{L}}(\mathbf{x}+\mathbf{y})+f_{\mathcal{L}}(\mathbf{x}-\mathbf{y})\right) / 2 \\
\underset{\mathbf{w} \sim D_{\mathcal{L}}}{\mathbb{E}}[\cos (2 \pi\langle\mathbf{w}, \mathbf{x}\rangle)]^{2} \underset{\mathbf{w} \sim D_{\mathcal{L}}}{\mathbb{E}}[\cos (2 \pi\langle\mathbf{w}, \mathbf{y}\rangle)]^{2} & \leq \underset{\mathbf{w} \sim D_{\mathcal{L}}}{\mathbb{E}}[\cos (2 \pi\langle\mathbf{w}, \mathbf{x}\rangle) \cos (2 \pi\langle\mathbf{w}, \mathbf{y}\rangle)]^{2} \\
-\underset{\mathbf{w} \sim D_{\mathcal{L}}}{\mathbb{E}}[\sin (2 \pi\langle\mathbf{w}, \mathbf{x}\rangle) \sin (2 \pi\langle\mathbf{w}, \mathbf{y}\rangle)]^{2} & \underset{\mathbf{w} \sim D_{\mathcal{L}}}{\mathbb{E}}[\cos (2 \pi\langle\mathbf{w}, \mathbf{x}\rangle)] \underset{\mathbf{w} \sim D_{\mathcal{L}}}{\mathbb{E}}[\cos (2 \pi\langle\mathbf{w}, \mathbf{y}\rangle)]
\end{aligned}
$$

Proof. Eq. (4a) follows from the definition of $f_{\mathcal{L}}$. Eq. (4b) follows from plugging in $\mathbf{y}=\mathbf{x}$ to Eq. (4a). Eq. (4c) follows from the fact that $\sqrt{a b} \leq(a+b) / 2$ for all $a, b \geq 0$. For Eq. (4d), use the Poisson summation formula to write $f_{\mathcal{L}^{*}}(\mathbf{x})$ in its dual form as

$$
f_{\mathcal{L}^{*}}(\mathbf{x})=\underset{\mathbf{w} \sim D_{\mathcal{L}}}{\mathbb{E}}[\cos (2 \pi\langle\mathbf{w}, \mathbf{x}\rangle)],
$$

where $\mathcal{L}^{*}$ is the dual lattice. We can then apply the identity $\cos (a+b)=\cos (a) \cos (b)-\sin (a) \sin (b)$ to derive Eq. (4d) from Eq. 4a). Finally, Eq. (4e) follows from applying the same analysis to (4c).

\section{Moments of the discrete Gaussian distribution}

We will need the Hessian product identity

$$
H(f(\mathbf{x}) g(\mathbf{x}))=f(\mathbf{x}) H g(\mathbf{x})+g(\mathbf{x}) H f(\mathbf{x})+\nabla f(\mathbf{x})(\nabla g(\mathbf{x}))^{T}+\nabla g(\mathbf{x})(\nabla f(\mathbf{x}))^{T} .
$$

We next show an inequality concerning the Hessian of $f_{\mathcal{L}}$. In particular, this inequality constrains the shape of the local maxima of $f_{\mathcal{L}}$. (As observed in [DRS14], $f_{\mathcal{L}}$ can in fact have local maxima at non-lattice points.) 
Proposition 3.1. For any lattice $\mathcal{L} \subset \mathbb{R}^{n}$ and any vector $\mathbf{x} \in \mathbb{R}^{n}$, we have the positive semidefinite inequality

$$
\frac{H f_{\mathcal{L}}(\mathbf{x})}{f_{\mathcal{L}}(\mathbf{x})} \succeq H f_{\mathcal{L}}(\mathbf{0})+\frac{\nabla f_{\mathcal{L}}(\mathbf{x})\left(\nabla f_{\mathcal{L}}(\mathbf{x})\right)^{T}}{f_{\mathcal{L}}(\mathbf{x})^{2}} .
$$

Proof. By Eq. (4a), we have

$$
f_{\mathcal{L}}(\mathbf{x}+\mathbf{y}) f_{\mathcal{L}}(\mathbf{x}-\mathbf{y})-f_{\mathcal{L}}(\mathbf{x})^{2} f_{\mathcal{L}}(\mathbf{y})^{2} \geq 0 .
$$

Note that we have equality when $\mathbf{y}=\mathbf{0}$. It follows that, for any $\mathbf{x}$, the left-hand side has a local minimum at $\mathbf{y}=\mathbf{0}$, and therefore the Hessian with respect to $\mathbf{y}$ at $\mathbf{0}$ must be positive semidefinite. The result follows by using Eq. (5) to take the Hessian and rearranging.

As a corollary, we obtain that the covariance matrix of $D_{\mathcal{L}+\mathbf{x}}$ is minimized at $\mathbf{x}=\mathbf{0}$. (Notice that the expectation of the centered Gaussian $D_{\mathcal{L}}$ is zero because the lattice is symmetric.) The corollary follows immediately from Proposition 3.1 and the following two identities:

$$
\begin{aligned}
& \frac{\nabla f_{\mathcal{L}}(\mathbf{x})}{f_{\mathcal{L}}(\mathbf{x})}=\frac{\nabla \rho(\mathcal{L}+\mathbf{x})}{\rho(\mathcal{L}+\mathbf{x})}=-2 \pi \underset{\mathbf{w} \sim D_{\mathcal{L}+\mathbf{x}}}{\mathbb{E}}[\mathbf{w}] \text {, and } \\
& \frac{H f_{\mathcal{L}}(\mathbf{x})}{f_{\mathcal{L}}(\mathbf{x})}=\frac{H \rho(\mathcal{L}+\mathbf{x})}{\rho(\mathcal{L}+\mathbf{x})}=4 \pi^{2} \underset{\mathbf{w} \sim D_{\mathcal{L}+\mathbf{x}}}{\mathbb{E}}\left[\mathbf{w} \mathbf{w}^{T}\right]-2 \pi I_{n} .
\end{aligned}
$$

Corollary 3.2. For any lattice $\mathcal{L} \subset \mathbb{R}^{n}$ and vector $\mathbf{x} \in \mathbb{R}^{n}$, we have the positive semidefinite inequality

$$
\underset{\mathbf{w} \sim D_{\mathcal{L}+\mathbf{x}}}{\mathbb{E}}\left[\mathbf{w} \mathbf{w}^{T}\right]-\underset{\mathbf{w} \sim D_{\mathcal{L}+\mathbf{x}}}{\mathbb{E}}[\mathbf{w}] \underset{\mathbf{w} \sim D_{\mathcal{L}+\mathbf{x}}}{\mathbb{E}}\left[\mathbf{w}^{T}\right] \succeq \underset{\mathbf{w} \sim D_{\mathcal{L}}}{\mathbb{E}}\left[\mathbf{w} \mathbf{w}^{T}\right]
$$

In particular,

$$
\underset{\mathbf{w} \sim D_{\mathcal{L}+\mathbf{x}}}{\mathbb{E}}\left[\|\mathbf{w}\|^{2}\right]-\left\|\underset{\mathbf{w} \sim D_{\mathcal{L}+\mathbf{x}}}{\mathbb{E}}[\mathbf{w}]\right\|^{2} \geq \underset{\mathbf{w} \sim D_{\mathcal{L}}}{\mathbb{E}}\left[\|\mathbf{w}\|^{2}\right]
$$

The following proposition (with $\mathbf{u}=\mathbf{v}$ ) implies that the one-dimensional projections of the discrete Gaussian distribution are "leptokurtic," i.e., have kurtosis at least 3, the kurtosis of a normal variable. We remark that the case $n=1$ follows from a known inequality related to the Riemann zeta function [Chu76, New76] (see also [BPY01, Section 2.2]).

Proposition 3.3. For any lattice $\mathcal{L} \subset \mathbb{R}^{n}$ and vectors $\mathbf{u}, \mathbf{v} \in \mathbb{R}^{n}$,

$$
\underset{\mathbf{y} \sim D_{\mathcal{L}}}{\mathbb{E}}\left[\langle\mathbf{y}, \mathbf{u}\rangle^{2}\langle\mathbf{y}, \mathbf{v}\rangle^{2}\right] \geq \underset{\mathbf{y} \sim D_{\mathcal{L}}}{\mathbb{E}}\left[\langle\mathbf{y}, \mathbf{u}\rangle^{2}\right] \underset{\mathbf{y} \sim D_{\mathcal{L}}}{\mathbb{E}}\left[\langle\mathbf{y}, \mathbf{v}\rangle^{2}\right]+2 \underset{\mathbf{y} \sim D_{\mathcal{L}}}{\mathbb{E}}[\langle\mathbf{y}, \mathbf{u}\rangle\langle\mathbf{y}, \mathbf{v}\rangle]^{2} .
$$

Proof. From Corollary 3.2, we have

$$
\underset{\mathbf{w} \sim D_{\mathcal{L}+\mathbf{x}}}{\mathbb{E}}\left[\langle\mathbf{w}, \mathbf{u}\rangle^{2}\right]-\underset{\mathbf{w} \sim D_{\mathcal{L}+\mathbf{x}}}{\mathbb{E}}[\langle\mathbf{w}, \mathbf{u}\rangle]^{2}-\underset{\mathbf{w} \sim D_{\mathcal{L}}}{\mathbb{E}}\left[\langle\mathbf{w}, \mathbf{u}\rangle^{2}\right] \geq 0
$$

Note that the left-hand side equals zero when $\mathbf{x}=\mathbf{0}$ since $\mathcal{L}=-\mathcal{L}$. The same is true if we multiply through by $\rho(\mathcal{L}+\mathbf{x})^{2}$, which leads to the inequality

$$
\sum_{\mathbf{y}, \mathbf{y}^{\prime} \in \mathcal{L}} \rho(\mathbf{y}+\mathbf{x}) \rho\left(\mathbf{y}^{\prime}+\mathbf{x}\right) \cdot\left(\left\langle\mathbf{y}-\mathbf{y}^{\prime}, \mathbf{u}\right\rangle^{2} / 2-\underset{\mathbf{w} \sim D_{\mathcal{L}}}{\mathbb{E}}\left[\langle\mathbf{w}, \mathbf{u}\rangle^{2}\right]\right) \geq 0 .
$$


(To see that, use $\left\langle\mathbf{y}-\mathbf{y}^{\prime}, \mathbf{u}\right\rangle=\langle\mathbf{y}+\mathbf{x}, \mathbf{u}\rangle-\left\langle\mathbf{y}^{\prime}+\mathbf{x}, \mathbf{u}\right\rangle$, and expand the square.) Therefore, as in the proof of Proposition 3.1, the Hessian of the left-hand side with respect to $\mathbf{x}$ at $\mathbf{x}=\mathbf{0}$ must be positive semidefinite. Using Eqs. (5), (6), and (7), we see that

$$
\left.H\left(\rho(\mathbf{y}+\mathbf{x}) \rho\left(\mathbf{y}^{\prime}+\mathbf{x}\right)\right)\right|_{\mathbf{x}=\mathbf{0}}=4 \pi^{2} \rho(\mathbf{y}) \rho\left(\mathbf{y}^{\prime}\right)\left(\left(\mathbf{y}+\mathbf{y}^{\prime}\right)\left(\mathbf{y}+\mathbf{y}^{\prime}\right)^{T}-I_{n} / \pi\right) .
$$

Therefore,

$$
\begin{aligned}
0 & \preceq \underset{\mathbf{y}, \mathbf{y}^{\prime} \sim D_{\mathcal{L}}}{\mathbb{E}}\left[\left(\left(\mathbf{y}+\mathbf{y}^{\prime}\right)\left(\mathbf{y}+\mathbf{y}^{\prime}\right)^{T}-I_{n} / \pi\right) \cdot\left(\left\langle\mathbf{y}-\mathbf{y}^{\prime}, \mathbf{u}\right\rangle^{2} / 2-\underset{\mathbf{w} \sim D_{\mathcal{L}}}{\mathbb{E}}\left[\langle\mathbf{w}, \mathbf{u}\rangle^{2}\right]\right)\right] \\
& =\underset{\mathbf{y}, \mathbf{y}^{\prime} \sim D_{\mathcal{L}}}{\mathbb{E}}\left[\left(\mathbf{y}+\mathbf{y}^{\prime}\right)\left(\mathbf{y}+\mathbf{y}^{\prime}\right)^{T} \cdot\left(\left\langle\mathbf{y}-\mathbf{y}^{\prime}, \mathbf{u}\right\rangle^{2} / 2-\underset{\mathbf{w} \sim D_{\mathcal{L}}}{\mathbb{E}}\left[\langle\mathbf{w}, \mathbf{u}\rangle^{2}\right]\right)\right] \\
& =\underset{\mathbf{y}, \mathbf{y}^{\prime} \sim D_{\mathcal{L}}}{\mathbb{E}}\left[\mathbf{y} \mathbf{y}^{T}\langle\mathbf{y}, \mathbf{u}\rangle^{2}+\mathbf{y} \mathbf{y}^{T}\left\langle\mathbf{y}^{\prime}, \mathbf{u}\right\rangle^{2}-\left(\mathbf{y} \mathbf{y}^{\prime T}+\mathbf{y}^{\prime} \mathbf{y}^{T}\right)\langle\mathbf{y}, \mathbf{u}\rangle\left\langle\mathbf{y}^{\prime}, \mathbf{u}\right\rangle-2 \mathbf{y} \mathbf{y}^{T} \underset{\mathbf{w} \sim D_{\mathcal{L}}}{\mathbb{E}}\left[\langle\mathbf{w}, \mathbf{u}\rangle^{2}\right]\right] \\
& =\underset{\mathbf{y} \sim D_{\mathcal{L}}}{\mathbb{E}}\left[\mathbf{y} \mathbf{y}^{T}\langle\mathbf{y}, \mathbf{u}\rangle^{2}\right]-\underset{\mathbf{y} \sim D_{\mathcal{L}}}{\mathbb{E}}\left[\mathbf{y} \mathbf{y}^{T}\right] \underset{\mathbf{y} \sim D_{\mathcal{L}}}{\mathbb{E}}\left[\langle\mathbf{y}, \mathbf{u}\rangle^{2}\right]-2 \underset{\mathbf{y} \sim D_{\mathcal{L}}}{\mathbb{E}}[\mathbf{y}\langle\mathbf{y}, \mathbf{u}\rangle] \underset{\mathbf{y} \sim D_{\mathcal{L}}}{\mathbb{E}}\left[\mathbf{y}^{T}\langle\mathbf{y}, \mathbf{u}\rangle\right],
\end{aligned}
$$

as needed.

\section{Monotonicity of the periodic Gaussian function}

The next proposition shows that $f_{\mathcal{L}, s}(\mathbf{x})$ is non-decreasing as a function of $s$. This (and the more general statement in Proposition 4.2] answers a question of Price [Pri14b], who proved it for the one-dimensional case $n=1$ (illustrated in Figure 2).

One might wonder if such a monotonicity property is specific to flat tori or whether it is a special case of a more general phenomenon. Namely, Peres [Per13] asked whether for any vertex transitive graph $G$ it holds that for any two vertices $u, v$, the ratio $\operatorname{Pr}\left[X_{t}=v\right] / \operatorname{Pr}\left[X_{t}=u\right]$ is non-decreasing as a function of $t$, where $X_{t}$ is a continuous-time random walk on $G$ starting at $u$ after time $t$. Recently, using our result, Price showed how to prove this for Abelian Cayley graphs [Pri16]. Interestingly, a further extension to arbitrary Cayley graphs turns out to be false [RS16a].

Proposition 4.1. For any lattice $\mathcal{L} \subset \mathbb{R}^{n}$ and vector $\mathbf{x} \in \mathbb{R}^{n}$,

$$
\frac{\frac{d}{d s} f_{\mathcal{L}, s}(\mathbf{x})}{f_{\mathcal{L}, s}(\mathbf{x})} \geq \frac{s}{2 \pi} \cdot \frac{\left\|\nabla f_{\mathcal{L}, s}(\mathbf{x})\right\|^{2}}{f_{\mathcal{L}, s}(\mathbf{x})^{2}} .
$$

Proof. A straightforward computation shows that

$$
\begin{aligned}
\frac{d}{d s} f_{\mathcal{L}, s}(\mathbf{x}) & =\frac{2 \pi f_{\mathcal{L}, s}(\mathbf{x})}{s^{3}} \cdot \underset{\mathbf{w} \sim D_{\mathcal{L}+\mathbf{x}, s}}{\mathbb{E}}\left[\|\mathbf{w}\|^{2}\right]-\frac{2 \pi f_{\mathcal{L}, s}(\mathbf{x})}{s^{3}} \cdot \underset{\mathbf{w} \sim D_{\mathcal{L}, s}}{\mathbb{E}}\left[\|\mathbf{w}\|^{2}\right] \\
& \geq \frac{2 \pi f_{\mathcal{L}, s}(\mathbf{x})}{s^{3}}\left\|\underset{\mathbf{w} \sim D_{\mathcal{L}+\mathbf{x}, s}}{\mathbb{E}}[\mathbf{w}]\right\|^{2},
\end{aligned}
$$

where we have applied Corollary 3.2. The result then follows from the fact that (see Eq.(6))

$$
\frac{\nabla f_{\mathcal{L}, s}(\mathbf{x})}{f_{\mathcal{L}, s}(\mathbf{x})}=-\frac{2 \pi}{s^{2}} \cdot \underset{\mathbf{w} \sim D_{\mathcal{L}+\mathbf{x}, s}}{\mathbb{E}}[\mathbf{w}]
$$




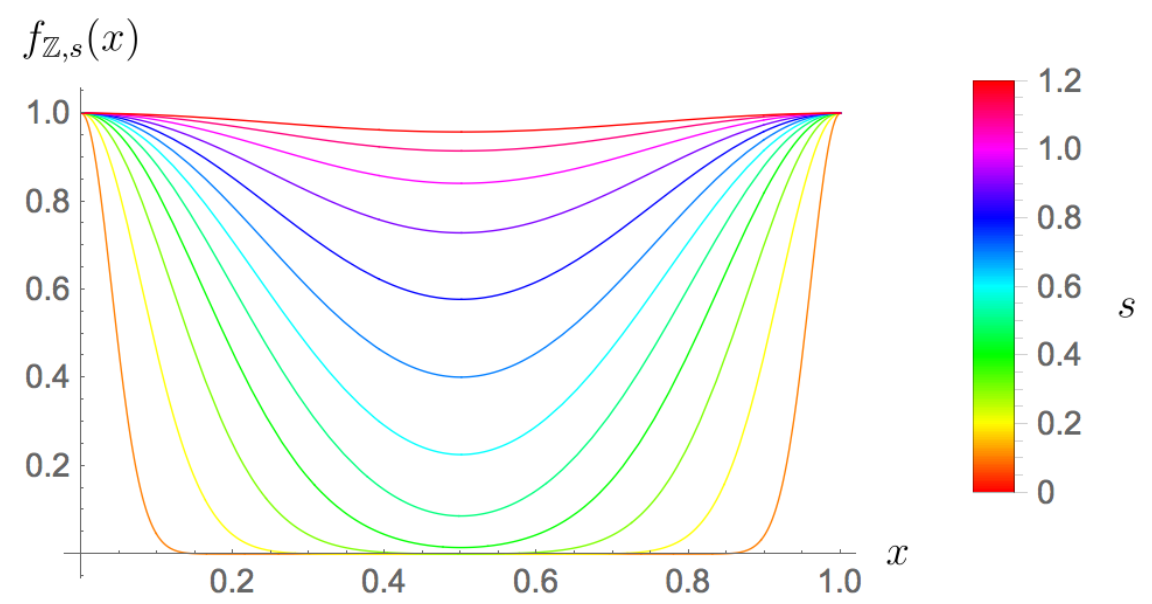

Figure 2: $f_{\mathbb{Z}, s}(x)$ for various values of $s$ and $x \in[0,1]$.

We now extend this monotonicity result by replacing the scalar variance parameter $s^{2}$ by a positive-definite matrix $\Sigma$. In particular, we define

$$
f_{\mathcal{L}, \Sigma}(\mathbf{x})=\frac{\sum_{\mathbf{y} \in \mathcal{L}} \exp \left(-\pi(\mathbf{y}+\mathbf{x})^{T} \Sigma^{-1}(\mathbf{y}+\mathbf{x})\right)}{\sum_{\mathbf{y} \in \mathcal{L}} \exp \left(-\pi \mathbf{y}^{T} \Sigma^{-1} \mathbf{y}\right)} .
$$

Equivalently,

$$
f_{\mathcal{L}, \Sigma}(\mathbf{x})=f_{\Sigma^{-1 / 2} \mathcal{L}}\left(\Sigma^{-1 / 2} \mathbf{x}\right)
$$

where $\Sigma^{1 / 2}$ is the unique positive-definite square root of $\Sigma$.

Proposition 4.2. For any lattice $\mathcal{L} \subset \mathbb{R}^{n}, \mathbf{x} \in \mathbb{R}^{n}$, and positive-definite matrices $\Sigma, \Sigma^{\prime} \in \mathbb{R}^{n \times n}$ satisfying the positive semidefinite inequality $\Sigma^{\prime} \preceq \Sigma$,

$$
f_{\mathcal{L}, \Sigma^{\prime}}(\mathbf{x}) \leq f_{\mathcal{L}, \Sigma}(\mathbf{x}) .
$$

Proof. We may replace $\mathcal{L}$ by $\Sigma^{\prime-1 / 2} \mathcal{L}$, $\mathbf{x}$ by $\Sigma^{\prime-1 / 2} \mathbf{x}$, and $\Sigma$ by $\Sigma^{\prime-1 / 2} \Sigma \Sigma^{\prime-1 / 2}$ so that we can assume without loss of generality that $\Sigma^{\prime}=I_{n}$. Moreover, by a change of basis, we may take $\Sigma$ to be diagonal. (Here, we have used the fact that the Gaussian is invariant under orthogonal transformations.)

So, it suffices to show that $f_{\mathcal{L}}(\mathbf{x}) \leq f_{\mathcal{L}, \Sigma}(\mathbf{x})$ when $\Sigma \in \mathbb{R}^{n \times n}$ is a diagonal matrix with $\Sigma \succeq I_{n}$. Let $s_{1}^{2}, \ldots, s_{n}^{2} \geq 1$ be the entries along the diagonal of $\Sigma$. The proof now proceeds nearly identically to the proof of Proposition 4.1. Differentiating with respect to $s_{i}$, we have

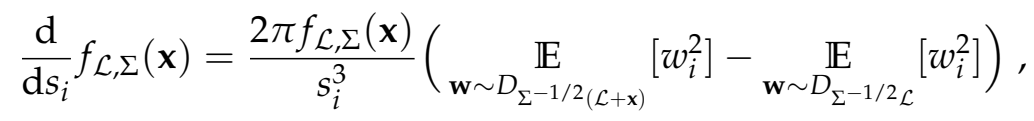

where $w_{i}$ is the $i$ th coordinate of $\mathbf{w}$. The result follows by noting that Corollary 3.2 implies that this derivative is positive for all $s_{i}>0$, so that $f_{\mathcal{L}, \Sigma}(\mathbf{x})$ is an increasing function of $s_{i}$.

We next give another monotonicity result, now with respect to taking sublattices. 
Proposition 4.3. For any lattice $\mathcal{L} \subset \mathbb{R}^{n}$, sublattice $\mathcal{M} \subseteq \mathcal{L}$, and vector $\mathbf{x} \in \mathbb{R}^{n}$,

$$
f_{\mathcal{M}}(\mathbf{x}) \leq f_{\mathcal{L}}(\mathbf{x})
$$

Proof.

$$
\begin{aligned}
\rho(\mathcal{M}+\mathbf{x}) \rho(\mathcal{L}) & =\sum_{\mathbf{c} \in \mathcal{L} / \mathcal{M}} \rho(\mathcal{M}+\mathbf{x}) \rho(\mathcal{M}+\mathbf{c}) \\
& \leq \sum_{\mathbf{c} \in \mathcal{L} / \mathcal{M}} \rho(\mathcal{M})(\rho(\mathcal{M}+\mathbf{x}+\mathbf{c})+\rho(\mathcal{M}+\mathbf{x}-\mathbf{c})) / 2 \\
& =\sum_{\mathbf{c} \in \mathcal{L} / \mathcal{M}} \rho(\mathcal{M}) \rho(\mathcal{M}+\mathbf{x}+\mathbf{c}) \\
& =\rho(\mathcal{M}) \rho(\mathcal{L}+\mathbf{x}) .
\end{aligned}
$$

The result follows.

\section{Positive correlation of the Gaussian measure on lattices}

The following shows that sublattices are positively correlated under the normalized Gaussian measure on a lattice. (Price asked whether this holds in the special case when $\mathcal{N}:=\mathcal{L} \cap V$ for some subspace $V \subseteq \mathbb{R}^{n}[$ Pri14a].)

Theorem 5.1. For any lattice $\mathcal{L} \subset \mathbb{R}^{n}$ and sublattices $\mathcal{M}, \mathcal{N} \subseteq \mathcal{L}$,

$$
\frac{\rho(\mathcal{M})}{\rho(\mathcal{L})} \cdot \frac{\rho(\mathcal{N})}{\rho(\mathcal{L})} \leq \frac{\rho(\mathcal{M} \cap \mathcal{N})}{\rho(\mathcal{L})}
$$

Proof. Note that the natural mapping from $\mathcal{M} /(\mathcal{M} \cap \mathcal{N})$ to $\mathcal{L} / \mathcal{N}$ given by $\mathbf{c} \mapsto \mathcal{N}+\mathbf{c}$ is injective. So,

$$
\begin{aligned}
\frac{\rho(\mathcal{L})}{\rho(\mathcal{N})} & =\sum_{\mathbf{c} \in \mathcal{L} / \mathcal{N}} \frac{\rho(\mathcal{N}+\mathbf{c})}{\rho(\mathcal{N})} \\
& \geq \sum_{\mathbf{c} \in \mathcal{M} /(\mathcal{M} \cap \mathcal{N})} \frac{\rho(\mathcal{N}+\mathbf{c})}{\rho(\mathcal{N})} \\
& \geq \sum_{\mathbf{c} \in \mathcal{M} /(\mathcal{M} \cap \mathcal{N})} \frac{\rho((\mathcal{M} \cap \mathcal{N})+\mathbf{c})}{\rho(\mathcal{M} \cap \mathcal{N})} \\
& =\frac{\rho(\mathcal{M})}{\rho(\mathcal{M} \cap \mathcal{N})} .
\end{aligned}
$$

The result follows by rearranging.

\section{Acknowledgements}

We thank Tom Price for helpful discussion, and the anonymous referees for their comments. 


\section{References}

[ADRS15] Divesh Aggarwal, Daniel Dadush, Oded Regev, and Noah Stephens-Davidowitz. Solving the shortest vector problem in $2^{n}$ time via discrete Gaussian sampling. In STOC, 2015. Available at http://arxiv.org/abs/1412.7994.

[ADS15] Divesh Aggarwal, Daniel Dadush, and Noah Stephens-Davidowitz. Solving the Closest Vector Problem in $2^{n}$ time- The discrete Gaussian strikes again! In FOCS, 2015. Available at http://arxiv.org/abs/1409.8063.

[Ban93] Wojciech Banaszczyk. New bounds in some transference theorems in the geometry of numbers. Mathematische Annalen, 296(4):625-635, 1993.

[BPY01] Philippe Biane, Jim Pitman, and Marc Yor. Probability laws related to the Jacobi theta and Riemann zeta functions, and Brownian excursions. Bull. Amer. Math. Soc. (N.S.), 38(4):435-465, 2001.

[Chu76] Kai Lai Chung. Excursions in Brownian motion. Ark. Mat., 14(2):155-177, 1976.

[Dad13] Daniel Dadush. Private communication, 2013.

[DRS14] Daniel Dadush, Oded Regev, and Noah Stephens-Davidowitz. On the closest vector problem with a distance guarantee. In IEEE 29th Conference on Computational Complexity, pages 98-109, 2014. Available at http://arxiv.org/abs/1409.8063.

[GPV08] Craig Gentry, Chris Peikert, and Vinod Vaikuntanathan. Trapdoors for hard lattices and new cryptographic constructions. In STOC, pages 197-206, 2008.

[HR13] Ishay Haviv and Oded Regev. The Euclidean distortion of flat tori. J. Topol. Anal., 5(2):205-223, 2013.

[MR07] Daniele Micciancio and Oded Regev. Worst-case to average-case reductions based on Gaussian measures. SIAM J. Comput., 37(1):267-302 (electronic), 2007.

[Mum07] David Mumford. Tata lectures on theta. I. Modern Birkhäuser Classics. Birkhäuser Boston, Inc., Boston, MA, 2007. With the collaboration of C. Musili, M. Nori, E. Previato and M. Stillman, Reprint of the 1983 edition.

[New76] Charles M. Newman. Fourier transforms with only real zeros. Proc. Amer. Math. Soc., 61(2):245-251 (1977), 1976.

[Per13] Yuval Peres, 2013. Personal communication.

[Pri14a] Thomas McMurray Price. Inequality regarding sum of gaussian on lattices. MathOverflow, 2014. http://mathoverflow . net/q/160507 (version: 2014-12-01).

[Pri14b] Thomas McMurray Price. Is the heat kernel more spread out with a smaller metric? MathOverflow, 2014. http://mathoverflow.net/q/186428 (version: 2014-12$11)$. 
[Pri15] Thomas McMurray Price. Numerical cohomology, 2015. http://arxiv.org/abs/ 1509.05797.

[Pri16] Thomas McMurray Price. An inequality for the heat kernel on an Abelian Cayley graph, 2016. https://arxiv.org/abs/1612.07306.

[Rie57] Bernhard Riemann. Theorie der Abel'schen Functionen. Journal für die reine und angewandte Mathematik, 54:101-155, 1857.

[Roy14] Thomas Royen. A simple proof of the Gaussian correlation conjecture extended to some multivariate gamma distributions. Far East J. Theor. Stat., 48(2):139-145, 2014.

[RS16a] Oded Regev and Igor Shinkar. A counterexample to monotonicity of relative mass in random walks. Electronic Communications in Probability, 2016. To appear. Available at http://arxiv.org/abs/1506.08631.

[RS16b] Oded Regev and Noah Stephens-Davidowitz. A Reverse Minkowski Theorem, 2016. https://arxiv.org/abs/1611.05979. 